PROCEEDINGS OF THE

AMERICAN MATHEMATICAL SOCIETY

Volume 138, Number 2, February 2010, Pages 567-575

S 0002-9939(09)10054-0

Article electronically published on September 16, 2009

\title{
A NOTE ON THE POINCARÉ INEQUALITY FOR LIPSCHITZ VECTOR FIELDS OF STEP TWO
}

\author{
MARIA MANFREDINI
}

(Communicated by Matthew J. Gursky)

\begin{abstract}
We provide a Poincaré inequality for families of Lipschitz continuous vector fields satisfying a Hörmander-type condition of step two.
\end{abstract}

\section{INTRODUCTION}

Given a family of vector fields $X_{1}, \ldots, X_{m}$ on $\mathbb{R}^{n}$, it is well known that the Poincaré inequality plays a crucial role when dealing with the regularity properties for operators of the form $\sum_{i=1}^{m} X_{i}^{2}$, in the setting of the first order Sobolev space related to the vector fields. In particular, the Poincaré inequality is the main tool in the Moser iteration technique for the study of the regularity of the solutions; see e.g. 4, 14, 5, 9, 3.

If the vector fields are smooth and satisfy the Hörmander condition (see [6])

$$
\operatorname{rank}\left(\operatorname{Lie}\left(X_{1}, \ldots, X_{m}\right)\right)(x)=n \text {, for all } x \in \mathbb{R}^{n},
$$

then the problem is quite well understood: the doubling property of the related control balls and the Poincaré inequality have been proved in [12] and [7] respectively.

The purpose of this paper is to give a contribution in the direction of finding other and different conditions assuring the Poincaré inequality.

In the non-smooth case: in 2 2 the authors proved the Poincaré inequality in a low regularity situation for vector fields of diagonal form, i.e. $X_{i}=\lambda_{i}(x) \partial_{i}$, $i=1, \ldots, n$, and the $\lambda_{i}$ 's were required to satisfy some strong condition (a strong form of a reverse Hölder inequality involving integral curves of vector fields). In [8] the authors do not need the smoothness of the vector fields, but they require that the control balls are representable by a controllable almost exponential maps introduced in [11]. In the recent paper [10] the Poincaré inequality is proved by developing the method of $[8$ for non-smooth and non-diagonal vector fields of step two, assuming the Lipschitz condition on the vector fields and on their commutators.

The fundamental results of the citated paper [12] about the study of the CarnotCarathéodory balls have been generalized to the two-parameter setting in [16] and to the multi-parameter setting in [15. These results are relevant for developing a theory of the multi-parameter singular integrals.

Received by the editors August 30, 2008.

2000 Mathematics Subject Classification. Primary 35A08, 35H20, 43A80; Secondary 35A17, 35J70.

(C)2009 American Mathematical Society Reverts to public domain 28 years from publication 
In many situations the regularity assumptions in [10] on the commutators are not satisfied, and it does not seem to be enough to get the Poincaré inequality.

Our interest in the question studied in this paper has been motivated by recent works on the regularity properties of some non-linear elliptic degenerate equations.

Consider, as a first example, the study of the Lipschitz minimal intrinsic graphs in the Heisenberg group $\mathbb{H}^{n}$ of dimension $n>1$; see [1]. In this setting an intrinsic regular surface can be represented as a graph of a Lipschitz continuous function in the euclidean sense $u: \Omega \subset \mathbb{R}^{2 n} \rightarrow \mathbb{R}$. The prescribed mean curvature equation for intrinsic graphs has the following expression:

$$
\sum_{i=1}^{2 n-1} X_{i}\left(\frac{X_{i} u}{\sqrt{1+|\nabla u|^{2}}}\right)=f, \text { in } \Omega \subset \mathbb{R}^{2 n},
$$

where

$$
\begin{gathered}
X_{i}=\partial_{i}, \text { for } i=1, \ldots, n-1, \\
X_{i}=\partial_{i}-x_{i-n+1} \partial_{2 n}, \text { for } i=n, \ldots, 2 n-2, \\
\quad X_{2 n-1}=\partial_{2 n-1}+u(x) \partial_{2 n},
\end{gathered}
$$

$\nabla u=\left(X_{1} u, \ldots, X_{2 n-1} u\right)$ and $|\cdot|$ denotes the euclidean norm. In particular, the vector field $X_{2 n-1}$ is non-linear, since it depends on the function $u$. We compute explicitly the commutators (in the distributional sense): if $k \leq n-1$ and $i=k+n-1$ or $i \leq n-1$ and $k=i+n-1$, then

$$
\left[X_{k}, X_{i}\right]=\operatorname{sign}(k-i) \partial_{2 n}
$$

if $i=2 n-1$ and $k<2 n-1$, then

$$
\left[X_{k}, X_{i}\right]=X_{k} u \partial_{2 n}
$$

if $k=2 n-1$ and $i \neq 2 n-1$, then

$$
\left[X_{k}, X_{i}\right]=-X_{i} u \partial_{2 n} .
$$

All other commutators vanish. Then, the vector fields $X_{1}, \ldots, X_{2 n-1}$ satisfy Hörmander's finite rank condition in $\mathbb{R}^{2 n}$ of order two; i.e. their commutators of length at most two span the tangent space at every point. Nevertheless, due to the minimal regularity of the coefficients (the function $u$ is only a euclidean Lipschitz continuous function), the result of [10] cannot be applied in this setting since the authors required that the commutators be Lipschitz continuous.

As a second example, we consider the situation illustrated in [13, where the regularity of the solutions of the subelliptic Monge-Ampére equation, det $D^{2} u=$ $k(x, u, \nabla u)$, are studied. The authors assume that the function $k$ has the form $k(x, r, \rho)=\sum_{i=1}^{N} p_{i}(x, r, \rho)^{2}$, where $p_{i}(x, r, \rho)$ are smooth functions on $\Omega \times \mathbb{R} \times \mathbb{R}^{n}$ locally satisfying $p_{i}(x, r, \rho) \approx|x|^{2}$. They introduce an adaptation of the Campanato methods, due to [17, to apply it to a sum of squares of rough vector fields arising in the partial transform system. More precisely, after an application of the partial Legendre trasform, the study of the Monge-Ampére equation is equivalent to the study of a problem of the type $\sum_{i, j=1}^{m} X_{i}^{*}\left(a_{i, j} X_{j} u\right)=f$ where $X_{1}, \ldots, X_{m}$ is an enumeration of the vector fields on $\mathbb{R}^{n}$ :

$$
X_{1}=\partial_{s}, \quad X_{\beta}^{1}=s \partial_{t_{\beta}}, X_{\beta}^{\alpha}=v_{\alpha}(s, t) \partial_{t_{\beta}}, 2 \leq \alpha, \beta \leq n,(s, t) \in \mathbb{R} \times \mathbb{R}^{n-1} .
$$

The functions $v_{\alpha}(s, t)$ are only Lipschitz continuous, and $\left\{a_{i, j}\right\}_{i, j=1}^{m}$ is a suitable constant matrix. The vector fields and their first order commutators span the 
tangent space $\mathbb{R}^{n}$ at every point in $\Omega$. But the system $\left\{X_{1}, \ldots, X_{m}\right\}$ does not have the regularity required in [10; then also in this setting, the result of [10] cannot be directly applied.

The aim of this paper is to fill this gap. The proof of the Poincaré inequality (also in the non-smooth setting) is based on Nagel-Stein-Weinger's lemma, which asserts, roughly speaking, that if $I=\left(i_{1}, \ldots, i_{n}\right)$ is an $n$-tuple of vector fields and commutators of order two, satisfying $\lambda_{I}(x) \neq 0$ and

$$
\left|\lambda_{I}(x)\right| r^{d(I)}>\frac{1}{2} \max _{J \in\{1, \ldots, q\}^{n}}\left|\lambda_{J}(x)\right| r^{d(J)}
$$

(see (2.6) and Section 2 for the definitions of $\lambda_{I}$ and $d(I)$ ), then the corresponding exponential map $E_{I}(x, \cdot)$ is one-to-one and the control balls are equivalent to the images of the exponential map of the ball-boxes (see (2.3) ) related to $I$. The choice of the $n$-tuple $I$ in (1.6) is crucial.

We note that we can repeat all the proof in [10] of the Poincaré inequality, as soon as only the commutators involved in the condition (1.6) are Lipschitz continuous.

In the above two examples, there exists an $n$-tuple $\bar{I}$ of vector fields and of their commutators of order two which are Lipschitz continuous, span $\mathbb{R}^{n}$, and where $\bar{I}$ satisfies the crucial condition (1.6). This fact suffices to prove the Poincaré inequality for the vector fields of the examples.

This is the plan of the paper. In Section 2 we recall some notation and known results. In Section 3 we introduce our conditions on the vector fields and prove the Poincaré inequality. Section 4 is devoted to the applications to the examples described above.

\section{Preliminary and knOWn Results}

For the reader's convenience we recall some notions. Given a family of locally Lipschitz continuous vector fields $X_{1}, \ldots, X_{m}$ on $\mathbb{R}^{n}$, we say that an absolutely continuous path $\gamma:[0, T] \rightarrow \mathbb{R}^{n}$ is a subunit if $\dot{\gamma}(t)=\sum_{i=1}^{m} b_{i}(t) X_{i}(\gamma(t))$, for almost all $t \in[0, T]$, with $\sum_{i=1}^{m}\left|b_{i}(t)\right|<1$. Assuming that for every $x_{1}, x_{2} \in \mathbb{R}^{n}$ there exists at least one subunit path connecting $x_{1}$ and $x_{2}$, the control distance or Carnot-Carathéodory distance is defined as

$$
\begin{aligned}
d\left(x_{1}, x_{2}\right)=\inf \left\{T>0: \exists \gamma:[0, T] \rightarrow \mathbb{R}^{n},\right. & \\
& \text { subunit path such that } \left.\gamma(0)=x_{1}, \gamma(T)=x_{2}\right\} .
\end{aligned}
$$

Denote by $B(x, r)$ the ball with a center at $x$ and radius $r$ with respect to the distance $d$.

We suppose that $X_{j}=\sum_{k=1}^{n} a_{j, k} \partial_{k}, j=1, \ldots, m$, are such that $a_{j, k}$ are locally Lipschitz continuous functions. We denote by $t \rightarrow e^{t X_{j}} x$ the integral curve of $X_{j}$ starting at $x$, and we assume that for any $x \in \mathbb{R}^{n}, j, k=1, \ldots, m$, the derivatives

$$
\left(X_{j} a_{k, j}-X_{k} a_{j, k}\right)(x)=\left.\frac{d}{d t}\left(a_{k, j}\left(e^{t X_{j}} x\right)-a_{j, k}\left(e^{t X_{k}} x\right)\right)\right|_{t=0}
$$

exist and the functions $X_{j} a_{j, k}-X_{k} a_{k, j}$ are continuous for all $j, k$. Denote the commutator by $\left[X_{j}, X_{k}\right]=\left\langle X_{j} a_{k, j}-X_{k} a_{j, k}, \nabla\right\rangle:=\sum_{i=1}^{n} b_{i}^{j, k} \partial_{i}$.

We assume that

$$
\operatorname{span}\left\{X_{j}(x),\left[X_{j}, X_{k}\right](x): j, k=1, \ldots, m\right\}=\mathbb{R}^{n} \quad \text { for any } x \in \mathbb{R}^{n} .
$$


We recall the definition of an almost exponential map related to a family of locally Lipschitz continuous vector fields introduced in [11. Given a pair of locally Lipschitz continuous vector fields $X$ and $Y$, define for small $s$ the map

$$
\exp ^{*}(s[X, Y]):=\left\{\begin{array}{cc}
e^{-\sqrt{s} Y} e^{-\sqrt{s} X} e^{\sqrt{s} Y} e^{\sqrt{s} X} x & \text { if } s \geq 0, \\
e^{-\sqrt{|s|} X} e^{-\sqrt{|s|} Y} e^{\sqrt{|s|} X} e^{\sqrt{|s|} Y} x & \text { if } s \leq 0 .
\end{array}\right.
$$

Roughly speaking, $\exp ^{*}(S)$ can be thought of as an approximate exponential of the commutator $S$.

We enumerate the vector fields and their first order brackets as

$$
Y_{j}=X_{j}, j=1, \ldots, m, \quad\left\{Y_{m+1}, \ldots, Y_{q}\right\}=\left\{\left[X_{i}, X_{j}\right], 1 \leq i<j \leq m\right\} .
$$

As usual, we attach to any fields $Y_{1}, \ldots, Y_{m}$ a degree one, to any commutators of order two a degree 2 , and we denote it by $d\left(Y_{i}\right)$.

Given an $n$-tuple of integer $I=\left(i_{1}, \ldots, i_{n}\right) \in\{1, \ldots, q\}^{n}$, define

$$
E_{I}(x, h)=\left(\exp ^{*}\left(h_{1} Y_{i_{1}}\right) \circ \cdots \circ \exp ^{*}\left(h_{n} Y_{i_{n}}\right)\right)(x)
$$

where $\exp ^{*}\left(h_{j} Y_{i_{j}}\right)(x)=\exp \left(h_{j} Y_{i_{j}}\right)(x)$ if $i_{j} \in\{1, \ldots, m\}$. The map $E_{I}$ is a somewhat modified version of the exponential map used in [12, in the smooth case, in the proof of their representation result of the Carnot-Carathéodory balls. We refer to [11, 8] and [10, where the map $E_{I}$ has been studied in a non-smooth situation. We also quote [16] and [15, where the map $E_{I}$ has been defined and studied in the multi-parameter setting.

Define the ball box, Box $_{I}$, as

$$
\operatorname{Box}_{I}(x ; r)=\left\{x+\sum_{k=1}^{n} \xi_{k} Y_{i_{k}}(x),\|\xi\|_{I} \leq r\right\}, \quad\|\xi\|_{I}=\max _{j=1, \ldots, n}\left|\xi_{j}\right|^{\frac{1}{d\left(Y_{i_{j}}\right)}} .
$$

Set $d(I)=\sum_{j=1}^{n} d\left(Y_{i_{j}}\right)$ and

$$
\lambda_{I}(x)=\operatorname{det}\left[Y_{i_{1}}(x), \ldots, Y_{i_{n}}(x)\right] .
$$

The following theorem, proved in [10, slightly generalizes the well-known result of structure of the balls in 12 for the smooth case and relies on a careful estimate of the derivatives of the map $h \longmapsto E_{I}(x, \cdot)$.

Theorem 2.1 (Theorem 3.2 in [10]). Suppose that for any compact set $K \subset \mathbb{R}^{n}$ there is $L>0$ such that, for every $i, j, k$, the coefficients $b_{i}^{j, k}$ of the commutators satisfy

$$
\left|b_{i}^{j, k}(x)-b_{i}^{j, k}(y)\right| \leq L d(x, y), \text { for every } x, y \in K .
$$

Then, there is a neighborhood $V$ of the origin in $\mathbb{R}^{n}$ such that the map $E_{I}(x, \cdot)$ is Lipschitz continuous in $V$, for all $I$ and for any $x \in K$. Moreover, there are $r_{0}>0$, $\varepsilon_{0}, \varepsilon_{1}, \varepsilon_{2}, \varepsilon_{3}>0$, which depend also on $L$, such that if $x \in K, r<r_{0}$ and I satisfy

$$
\left|\lambda_{I}(x)\right| r^{d(I)}>\frac{1}{2} \max _{J \in\{1, \ldots, q\}^{n}}\left|\lambda_{J}(x)\right| r^{d(J)},
$$

then

(i) $\frac{1}{2}\left|\lambda_{I}(x)\right| \leq\left|\operatorname{det} \frac{\partial E_{I}}{\partial h}(x, h)\right| \leq 2\left|\lambda_{I}(x)\right|$ for almost all $h \in R^{n},\|h\|_{I} \leq \varepsilon_{0} r$;

(ii) the map $E_{I}(x, \cdot)$ is one-to-one on $\left\{\|h\|_{I} \leq \varepsilon_{0} r\right\}$;

(iii) $B\left(x, \varepsilon_{2} r\right) \subset B o x_{I}\left(x, \varepsilon_{1} r\right) \subset\left\{E_{I}(x, h):\|h\|_{I} \leq \varepsilon_{0} r\right\} \subset B\left(x, \varepsilon_{3} r\right)$. 
Theorem 2.1 gives the representation of the Carnot-Carathéodory balls by means of the maps $E_{I}$, which are controllable in the sense of [8]. As a consequence, the doubling property of the Lebesgue measure of the balls and the Poincaré inequality hold; see Theorem 1.2 in [10].

\section{Poincaré inequality}

In this section, we establish our version of the Poincaré inequality. Consider the vector fields $X_{j}=\sum_{k=1}^{n} a_{j, k} \partial_{k}, j=1, \ldots, m$, where the coefficients $a_{j, k}$ are locally Lipschitz continuous functions. Suppose also that they satisfy the Hörmander condition of step two (2.1).

We assume that

(H): Given a compact set $K \subset \mathbb{R}^{n}$, for every $x \in K$ there exists an $n$-tuple $\bar{I}=\left(i_{1}, \ldots, i_{n}\right) \in\{1, \ldots, q\}^{n}$ such that $\lambda_{\bar{I}}(x) \neq 0$ and

$$
\left|\lambda_{\bar{I}}(x)\right| r^{d(\bar{I})}>\frac{1}{2} \max _{J \in\{1, \ldots, q\}^{n}}\left|\lambda_{J}(x)\right| r^{d(J)} .
$$

Besides, we required that the coefficients of the commutators corresponding to the $n$-tuple $\bar{I}$ are locally Lipschitz continuous functions.

According to the enumeration in (2.2), the $q$ vector fields $Y_{1}, \ldots, Y_{q}$ are in general $q>n$. Then, at each point of $\mathbb{R}^{n}$ many different subsets of $\left\{Y_{1}, \ldots, Y_{q}\right\}$ form a basis for the tangent space to $\mathbb{R}^{n}$. One of the many difficulties is finding a good choice for a basis, as a subcollection of vector fields, $Y_{i_{1}}, \ldots, Y_{i_{n}}$ of the $Y_{1}, \ldots Y_{q}$ for performing calculations. A possible choice as a basis is $Y_{i_{1}}, \ldots, Y_{i_{n}}$, where the $\left(i_{1}, \ldots, i_{n}\right)$ is equal to $\bar{I}$ in condition $(\mathbf{H})$.

Theorem 3.1. Suppose that the vector fields satisfy $\mathbf{( H )}$. For every compact set $K$, there is a neighborhood $V$ of the origin in $\mathbb{R}^{n}$ such that the map $E_{\bar{I}}(x, \cdot)$ is Lipschitz continuous in $V$ for any $x \in K$. Moreover, there are $r_{0}>0, \varepsilon_{0}, \varepsilon_{1}, \varepsilon_{2}, \varepsilon_{3}>0$ such that if $x \in K, r<r_{0}$, then

(i) $\frac{1}{2}\left|\lambda_{\bar{I}}(x)\right| \leq\left|\operatorname{det} \frac{\partial E_{\bar{I}}}{\partial h}(x, h)\right| \leq 2\left|\lambda_{\bar{I}}(x)\right|$ for almost all $h \in R^{n},\|h\|_{\bar{I}} \leq \varepsilon_{0} r$;

(ii) the map $E_{\bar{I}}(x, \cdot)$ is one-to-one on $\left\{\|h\|_{\bar{I}} \leq \varepsilon_{0} r\right\}$;

(iii) $B\left(x, \varepsilon_{2} r\right) \subset \operatorname{Box}_{\bar{I}}\left(x, \varepsilon_{1} r\right) \subset\left\{E_{\bar{I}}(x, h):\|h\|_{\bar{I}} \leq \varepsilon_{0} r\right\} \subset B\left(x, \varepsilon_{3} r\right)$.

Proof. It is sufficient to repeat, step by step, the proof of Theorem 3.2 in [10] only for the selected $n$-tuple $\bar{I}$ in condition (H). Indeed, fix a compact set $K \subset \mathbb{R}^{n}$. Then by $(\mathbf{H})$, for every $x \in K$ there exists an $n$-tuple $\bar{I}=\left(i_{1}, \ldots, i_{n}\right) \in\{1, \ldots, q\}^{n}$ which satisfies (3.1) and such that the vector fields of the corresponding basis $Y_{i_{1}}, \ldots, Y_{i_{n}}$ are locally Lipschitz continuous.

In the following the $n$-tuple $\bar{I}$ satisfying condition (H) is fixed. Then, arguing as in Lemma 3.3 in [10], there exists a neighborhood $V$ of the origin in $\mathbb{R}^{n}$ such that the map $E_{\bar{I}}(x, \cdot)$ is Lipschitz continuous on $V$, with a Lipschitz norm which depends on the Lipschitz norm of the original vector fields $X_{1}, \ldots, X_{m}$ and of the involved selected family $Y_{i_{1}}, \ldots, Y_{i_{n}}$. Besides, the map $E_{\bar{I}}(x, \cdot)$ belongs to $W^{1, \infty}(V)$, and, as in [10], its (distributional) derivative $\partial_{h_{j}} E_{\bar{I}}(x, \cdot)$ is such that (at a point of differentiability of $\left.E_{\bar{I}}(x, \cdot)\right)$ for $j=1, \ldots, n$,

$$
\partial_{h_{j}} E_{\bar{I}}(x, h)=Y_{i_{j}}(x)+R_{j}(x, h) \text {, for a.e. } h \in V,
$$

where the remainder $R_{j}$ satisfies the estimate

$$
\left|R_{j}(x, h)\right| \leq C|| h \|_{\bar{I}}, \text { for any } h \in V .
$$


Besides, from Proposition 3.5 in [10] we can write for almost any $h$,

$$
\partial_{h_{j}} E_{\bar{I}}(x, h)=Y_{i_{j}}(x)+\sum_{k=1}^{n} b_{j, k}(x, h) Y_{i_{k}}(x),
$$

where $\left|b_{j, k}(x, h)\right| \leq c r^{d\left(Y_{i_{k}}\right)-d\left(Y_{i_{j}}\right)}$, for any $\|h\|_{\bar{I}}<\varepsilon_{0} r$. Then for a.e. $h$,

$$
\operatorname{det} \partial_{h_{j}} E_{\bar{I}}(x, h)=\lambda_{\bar{I}}(x) \operatorname{det}\left(\delta_{j, k}+b_{j, k}\right)
$$

(here $\delta_{j, k}$ denotes the Kronecker symbol). The proof of $(i)$ can be easily concluded as in [10, using the above estimate of $\left|b_{j, k}(x, h)\right|$. Finally, by the same argument of [10, we can prove the injectivity of the map $E_{\bar{I}}(x, \cdot)$ in assertion $(i i)$ and the equivalence in $($ iii).

Then, as a consequence of the representation result of the balls in Theorem 3.1 , the doubling property and the Poincaré inequality hold:

Theorem 3.2. Suppose that the vector fields satisfy $(\mathbf{H})$. For any compact set $K \subset \mathbb{R}^{n}$, there are $c, r_{0}, Q>0, \rho \geq 1$, depending on $K$, such that

$$
|B(x, 2 r)| \leq 2^{Q}|B(x, r)|, \text { for all } x \in K, r<r_{0}
$$

and

$$
\int_{B(x, r)}\left|u(y)-u_{B}\right| d y \leq c r \int_{B(x, \rho r)}|\nabla u(y)| d y, \text { for all } u \in C^{1}(B(x, \rho r))
$$

where $u_{B}=\frac{1}{|B|} \int_{B} u$.

Proof. The doubling property of the balls follows immediately by (iii) in Theorem 3.1 and by condition (3.1) in $(\mathbf{H})$. The arguments to prove the Poincaré inequality are the same as in the proof of Theorem 1.2 in [10] (for the selected $n$-tuple $\bar{I}$ in condition $(\mathbf{H})$ ). It is sufficient to prove the hypotheses of Theorem 2.1 in 8 .

Letting

$$
\Omega_{\bar{I}}=\left\{x \in B\left(x_{0}, \varepsilon_{2} r\right):\left|\lambda_{\bar{I}}(x)\right| r^{d(\bar{I})}>\frac{1}{2} \max _{J \in\{1, \ldots, q\}^{n}}\left|\lambda_{J}(x)\right| r^{d(J)}\right\},
$$

then in the language of [8] the map $E_{\bar{I}}: \Omega_{\bar{I}} \times\left\{\|h\|_{\bar{I}} \leq \varepsilon_{0} r\right\} \rightarrow \mathbb{R}$ is an almost exponential map. The controllability in [8] requires that the points $x \in \Omega_{\bar{I}}$ and $E_{\bar{I}}(x, h)$ can be joined by a piecewise integral curve of the vector fields $X_{j}$. We refer to [10] for a detailed proof of this fact. We can conclude, as in [10], that all the hypotheses of Theorem 2.1 in $[8]$ are satisfied and then the Poincaré inequality holds.

\section{Some EXAmples}

In both cases of the examples in the introduction, the extra regularity properties of the commutators required in (2.5) are not satisfied. On the contrary, the condition $(\mathbf{H})$ is verified. 
4.1. Example 1. Consider the non-linear vector fields on $\mathbb{R}^{2 n}$ defined in (1.3) and (1.4). They satisfy Hörmander's finite rank condition of order two (2.1). We introduce a modification of the vector fields as follows. Let $c$ be a positive constant, which we choose later, and consider the vector fields

$$
\begin{aligned}
& X_{i}=c \partial_{i}, \text { for } i=1, \ldots, n-1, \\
& X_{i}=c \partial_{i}-c x_{i-n+1} \partial_{2 n}, \quad \text { for } i=n, \ldots, 2 n-2,
\end{aligned}
$$

and

$$
X_{2 n-1}=\partial_{2 n-1}+u(x) \partial_{2 n} .
$$

We recall that $u$ is a euclidean Lipschitz continuous function. If $k \leq n-1$ and $i=k+n-1$ or $i \leq n-1$ and $k=i+n-1$, then

$$
\left[X_{k}, X_{i}\right]=c^{2} \operatorname{sign}(k-i) \partial_{2 n} ;
$$

if $i=2 n-1$ and $k<2 n-1$, then

$$
\left[X_{k}, X_{i}\right]=c X_{k} u \partial_{2 n}
$$

if $k=2 n-1$ and $i \neq 2 n-1$, then

$$
\left[X_{k}, X_{i}\right]=-c X_{i} u \partial_{2 n} .
$$

All other commutators vanish.

We put $X_{2 n}:=\left[X_{n}, X_{1}\right]=c^{2} \operatorname{sign}(n-1) \partial_{2 n}$, and we choose the $2 n$-tuple

$$
\bar{I}=(1,2, \ldots, 2 n-1,2 n) \text {. }
$$

Then $d(\bar{I})=2 n+1$ and

$$
\left|\lambda_{\bar{I}}(x)\right|=\left|\operatorname{det}\left[X_{1}(x), \ldots, X_{2 n-1}(x), X_{2 n}(x)\right]\right|=c^{2 n+1} .
$$

Besides, if $J$ is an $2 n$-tuple such that $\lambda(J) \neq 0$, then $J$ is similar to $\bar{I}$, in the sense that the corresponding family of vector fields is of the type $\left(X_{1}, \ldots, X_{2 n-1}\right.$, $\left.c^{2} \operatorname{sign}(k-i) \partial_{2 n}\right)$ for some $k, i$; otherwise the corresponding family of vector fields is of the type $\left(X_{1}, \ldots, X_{2 n-1}, c X_{k} u \partial_{2 n}\right)$, for some $k$. In the first case $d(J)=2 n+1$ and $\left|\lambda_{J}(x)\right|=c^{2 n+1}$, in the second one $d(J)=2 n+1$ and

$$
\left|\lambda_{J}(x)\right|=c^{2 n}\left|X_{k} u(x)\right| \text {. }
$$

Then, for every compact set $K$ of $\mathbb{R}^{2 n}$, there exists a sufficiently large constant $c$ such that the $2 n$-tuple $\bar{I}$ satisfies condition (3.1). Indeed, if $c>\frac{1}{2} \sup _{K}\left|X_{k} u(x)\right|$ one has

$$
\left|\lambda_{\bar{I}}(x)\right| r^{d(\bar{I})}=c^{2 n+1} r^{2 n+1}>\frac{1}{2} c^{2 n}\left|X_{k} u(x)\right| r^{2 n+1} .
$$

4.2. Example 2. Consider the vector fields defined in (1.5). For simplicity of notation, we suppose $n=3$ and $(s, t)=\left(s, t_{2}, t_{3}\right) \in \mathbb{R}^{3}$. We enumerate the vector fields in (1.5) in the following way:

$$
\begin{gathered}
X_{1}:=c \partial_{s}, \quad X_{2}:=X_{2}^{1}=c s \partial_{t_{2}}, \quad X_{3}:=X_{3}^{1}=c s \partial_{t_{3}}, \\
X_{4}:=X_{2}^{2}=v_{2}(s, t) \partial_{t_{2}}, X_{5}:=X_{3}^{2}=v_{2}(s, t) \partial_{t_{3}}, \\
X_{6}:=X_{2}^{3}=v_{3}(s, t) \partial_{t_{2}}, \quad X_{7}:=X_{2}^{3}=v_{3}(s, t) \partial_{t_{3}},
\end{gathered}
$$

where the functions $v_{i}(s, t)$ are only Lipschitz continuous and $c$ is a positive constant which we choose later. We set

$$
X_{8}:=\left[X_{1}, X_{2}\right]=c^{2} \partial_{t_{2}}, \quad X_{9}:=\left[X_{1}, X_{3}\right]=c^{2} \partial_{t_{3}} .
$$


We enumerate all the other commutators of order two as $X_{10}, \ldots, X_{q}$.

Let $K$ be a compact set in $\mathbb{R}^{3}$. For every $(s, t) \in K$ there exists at least an 3tuple $I$ satisfying condition (3.1). If $I=\left(i_{1}, i_{2}, i_{3}\right)$ is such that all the corresponding vector fields $\left(X_{i_{1}}, X_{i_{2}}, X_{i_{3}}\right)$ have regular coefficients, that is, they do not contain derivatives of $v_{2}$ and $v_{3}$, then hypothesis $(\mathbf{H})$ is satisfied.

Otherwise, if for example

$$
\left(X_{i_{1}}, X_{i_{2}}, X_{i_{3}}\right)=\left(X_{1}, X_{2}, c s \partial_{t_{k}} v_{j}(s, t) \partial_{t_{3}}\right), d(I)=4
$$

for some $k, j$, then it is possible to choose $c$ sufficiently large in such a way that for $\bar{I}=(1,2,9), d(\bar{I})=4$, one has

$$
\left|\lambda_{\bar{I}}(s, t)\right| r^{d(\bar{I})}=c^{4} r^{4} \geq\left|\lambda_{I}(s, t)\right| r^{d(I)}=c^{3}\left|s \partial_{t_{k}} v_{j}(s, t)\right| r^{4}
$$

(i.e. $\left.c>\sup _{K}\left|s \partial_{t_{k}} v_{j}(s, t)\right|\right)$. If

$$
\left(X_{i_{1}}, X_{i_{2}}, X_{i_{3}}\right)=\left(X_{1}, X_{2}, c \partial_{s} v_{j}(s, t) \partial_{t_{3}}\right), d(I)=4
$$

for some $j$, then we can again choose $\bar{I}=(1,2,9)$. If

$$
\left(X_{i_{1}}, X_{i_{2}}, X_{i_{3}}\right)=\left(X_{1}, c s \partial_{t_{i}} v_{l}(s, t) \partial_{t_{2}}, c s \partial_{t_{k}} v_{j}(s, t) \partial_{t_{3}}\right), d(I)=5
$$

for some $i, l, k, j$, then it is possible to choose $c$ sufficiently large such that if $\bar{I}=$ $(1,8,9)$ (i.e. $\left(X_{1}, c^{2} \partial_{t_{2}}, c^{2} \partial_{t_{3}}\right)$ and $\left.d(\bar{I})=5\right)$, one has

$$
\left|\lambda_{\bar{I}}(s, t)\right| r^{d(\bar{I})}=c^{5} r^{5} \geq\left|\lambda_{I}(s, t)\right| r^{d(I)}=c^{3}\left|s^{2} \partial_{t_{i}} v_{l}(s, t) \partial_{t_{k}} v_{j}(s, t)\right| r^{5} \text {. }
$$

In all other cases we argue in the same way. Finally, for every $K$ compact set of $\mathbb{R}^{3}$, there is a sufficiently large constant $c$ such that condition $(\mathbf{H})$ is satisfied.

\section{REFERENCES}

1. L. Capogna, G. Citti, M. Manfredini, Smoothness of Lipschitz minimal intrinsic graphs in Heisenberg groups $\mathbb{H}^{n}, n>1$, preprint, to appear in Crelle's Journal.

2. B. Franchi, E. Lanconelli, Hölder regularity theorem for a class of linear nonuniformly elliptic operators with measurable coefficients, Ann. Scuola Norm. Sup. Pisa Cl. Sci. (4) 10 (1983), 523-541. MR753153 (85k:35094)

3. B. Franchi, G. Lu, R. Wheeden, A relationship between Poincaré type inequalities and representation formulas in spaces of homogeneous type, Internat. Math. Res. Notices, no. 1, (1996), 1-14. MR1383947 (97k:26012)

4. N. Garofalo, D.-M. Nhieu, Isoperimetric and Sobolev inequalities for Carnot-Carathéodory spaces and the existence of minimal surfaces, Comm. Pure Appl. Math. 49 (1996), 1081-1144. MR.1404326 (97i:58032)

5. P. Hajlasz, P. Koskela, Sobolev met Poincaré, Mem. Amer. Math. Soc. 145, no. 688 (2000). MR 1683160 (2000j:46063)

6. H. Hörmander, Hypoelliptic second order differential equations, Acta Math. 119 (1967), 147171. MR0222474 (36:5526)

7. D. Jerison, The Poincaré inequality for vector fields satisfying Hörmander's condition, Duke Math. J. 53 (1986), 503-523. MR850547 (87i:35027)

8. E. Lanconelli, D. Morbidelli, On the Poincaré inequality for vector fields, Ark. Mat. 38 (2000), 327-342. MR.1785405 (2002a:46037)

9. P. Maheux, L. Saloff-Coste, Analyse sur les boules d'un opérateur sous-elliptique, Math. Ann. 303 (1995), 713-740. MR 1359957 (96m:35049)

10. A. Montanari, D. Morbidelli, Balls defined by nonsmooth vector fields and the Poincaré inequality, Ann. Inst. Fourier (Grenoble) 54, no. 2 (2004), 431-452. MR.2073841 (2005e:46053)

11. D. Morbidelli, Fractional Sobolev norms and structure of the Carnot-Carathéodory balls for Hörmander vector fields, Studia Math. 139 (2000), 213-244. MR1762582 (2002a:46039)

12. A. Nagel, E. M. Stein, S. Wainger, Balls and metrics defined by vector fields. I: Basic properties, Acta Math. 155 (1985), 103-147. MR793239 (86k:46049) 
13. C. Rios, E. T. Sawyer, R. L. Wheeden, Regularity of subelliptic Monge-Ampère equations, Adv. Math. 217, no. 3 (2008), 967-1026. MR2383892

14. L. Saloff-Coste, A note on Poincaré, Sobolev and Harnack inequalities, Internat. Math. Res. Notices, no. 2 (1992), 27-38. MR.1150597 (93d:58158)

15. B. Street, Multi-parameter Carnot-Carathéodory balls and the theorem of Frobenius, preprint.

16. T. Tao, J. Wright, $L^{p}$ improving bounds for averages along curves, J. Amer. Math. Soc. 16 no. 3 (2003), 605-638. MR1969206 (2004j:42005)

17. C.-J. Xu, C. Zuily, Higher interior regularity for quasilinear subelliptic systems, Calc. Var. Partial Differential Equations 5 (1997), 323-343. MR.1450714 (98e:35039)

Dipartimento di Matematica, Università di Bologna, Piazza Porta S. Donato 5, 40126 Bologna, ItALY

E-mail address: manfredi@dm.unibo.it 